\section{Study Confirms High Eco-Efficiency of Integrated Processes}

Thesente he coating process is one of the most energy-intensive process steps in industrial automotive manufacturing. Volatile organic compounds (VOCs), energy consumption and $\mathrm{CO}_{2}$ balance play a major role. A recent comprehensive analysis performed by BASF and Dürr used a holistic perspective to examine the eco-efficiency of painting lines and coating technology. The eco-efficiency analysis developed by BASF looks at environmental impact in proportion to the cost-effectiveness of products and processes. Its aim is to help customers to decide which products and processes are the best choice, both ecologically and economically. To this end, the entire life cycle of a product or process is examined, from raw materials sourcing to product manufacture and use, as well as disposal. The most important results of the current study refer to coating processes with primers, shortened coating processes and the use of state-of-the-art painting line and application technologies. In processes with primers, BASF and Dürr studied the most important versions of solvent-borne and waterborne paint systems used in industry. The study found that that waterborne processes generally had good results overall in the evaluation of their ecoefficiency. A comparison of the eco-efficiency of conventional filler processes with that of integrated processes showed a clear advantage in favour of integrated processes. Significant potential for savings is yielded with respect to the energy consumption of the spray booths if they are operated with recirculating air. The study not only provides concrete figures. The comparability of the figures also allows realistic assumptions to be made on consumption, emissions and costs.

\title{
Eisenmann Sells E-Shuttle to Japan for the First Time
}

In the last days of 2012, Eisenmann secured an order worth millions from Isuzu for a dip-coating pretreatment plant. By investing in Eisenmann's E-Shuttle conveyor technology for dip-coating, the Japanese car maker will be able to uphold its commitment to continuous improvement. The new pre-treatment plant will come complete with control systems technology and electro dipcoating. A key factor in the purchase decision was in particular the flexibility of the Eisenmann system: the dip curves can be individually defined and the speeds configured for each type of vehicle, even in a mixed sequence. The Tokyo-based Japanese car maker will now replace its existing paint shop for truck cabs step by step. "This project gives us a valuable reference on the Japanese market and raises our profile," said Dr. Matthias von Krauland, Chairman of the Executive Board at Eisenmann AG. The E-Shuttle features three freely programma- ble horizontal, vertical and rotation axes, thus enabling all types of body to be transported through the pretreatment and electro dip-coating process with an individually defined dip curve that is precisely geared to each product's needs. "We were impressed by the high level of flexibility of this plant," said Yoshinobu Tamura, Senior Specialist, Paint Group, Isuzu. "Thanks to the lifting axis, which not only rotates but can also travel in a curve, we can define the ideal dip curves and the shortest process times individually for each body." Isuzu will produce 50 units per hour on the new plant, initially painting two different types of truck cab in a mixed sequence. Other body types can be added later. Assembly of the plant will begin this year, and the first cabs are expected to roll off the production line in March 2014. In total, 260 E-Shuttle dip-coating systems are currently deployed in Asia and Europe as well as in North and South America.
- Paint spray systems Special spray solutions for complex shapes Material tanks in many sizes and models

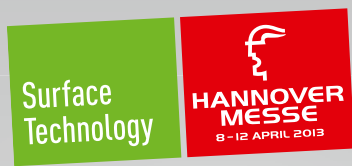

Let's meet: Hall 3, D13(9)

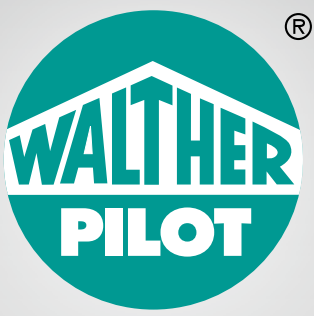

The Coatings Experts

WALTHER Spritz- und Lackiersysteme GmbH Kärntner Str. 18-30, D-42327 Wuppertal Phone +49(0)202 787-0 info@walther-pilot.de 